\title{
Review of MIMO-OFDM using Carrier Offset on behalf of Literature Survey
}

\author{
M.Tech.Scholar, Renu Dhankhar ${ }^{1}$, Mr. Parvinder Bangar ${ }^{2}$, Asst. Professor \\ Electronics and Communication Department, CBS Group Of Institution, Jhajjar, Haryana
}

\begin{abstract}
In this paper, proposed a joint estimation of Carrier Frequency Offset (CFO) and Channel Distortion for Multiple Input Multiple Output (MIMO) Orthogonal Frequency Division Multiplexing (OFDM) system using training sequences. Since the main objective of maximizing the sum of rates of all the nodes in the network can lead to a severe bias in rate allocation among the nodes. An important technique for an OFDM system is IFFT (Inverse Fast Fourier Transform) and FFT (Fast Fourier Transform) which is used for modulation and demodulation of OFDM systems. Carrier Frequency Offset (CFO) acquisition algorithm and Carrier Frequency Offset (CFO) tracking algorithm are proposed to improve the estimation accuracy of MIMO OFDM system. The proposed estimation method requires at least one OFDM training block for estimation and training symbols should be random Joint Estimation of Carrier Frequency Offset (CFO) and Channel Distortion Using Two Repeated Training Sequences m. If the OFDM training block consists of two repeated sequences, a low complexity two step approach used to solve any one of the two problems and important insights can be obtained by Inferring duality results for the other problem.
\end{abstract}

Keywords: Carrier-frequency offset (CFO), channel estimation, multiple-input multiple-output (MIMO), orthogonal frequency division multiplexing (OFDM)

\section{Introduction}

Recently, orthogonal frequency division multiplexing (OFDM) has become a wide spread wireless communication technique for transmission of signals through the wireless channels. The key challenge for future wireless communication systems is to provide high data rate access at high quality of service (QoS). Increase the spectral efficiency and improve link reliability. Multiple inputs multiple output (MIMO) OFDM wireless technology provides to meet these demands [1]. The maturing of MIMO OFDM technology leads to a several applications such as digital video broad casting (DVB), digital audio broadcasting (DAB), the IEEE 802.16a wireless metropolitan area network (WMAN) standard, and IEEE 802.11a wireless local area network (WLAN) standard. OFDM is also used for dedicated short-range communications and as a potential candidate for fourth generation (4G) mobile wireless communication systems [2]. In MIMO OFDM a collection of problems including carrier frequency offset (CFO), channel distortion and I/Q (in phase quadrature) imbalance can seriously degrade the system performance. Orthogonal frequency division multiplexing (OFDM) is a mostly useful modulation technique for transmission of signals over wireless communication channels. In OFDM systems the available bandwidth is utilized very efficiently without causing the inter-carrier interference (ICI). In MIMO OFDM systems multiple antennas have been used at both sides of the wireless link for interference cancellation and realize diversity gain.

The basic principle of an OFDM is dividing the available transmission bandwidth into several parallel narrowband channels. The input data is divided into several data streams or channels. Each channel modulated with a conventional modulation schemes such as quadrature amplitude modulation (QAM) or phase shift keying (PSK) [3]. In OFDM system the sub carriers are orthogonal to each other, So that the cross talk between the sub channels is eliminated and inter carrier guard bands are not required. OFDM orthogonally allows for efficient modulator and demodulator implementation using Fast Fourier Transform (FFT) algorithm on the receiver side and Inverse Fast Fourier Transform (IFFT) on the transmission side. The remained paper organized as follows. In section II, described a MIMO OFDM system model. In section III discussed the Carrier Frequency Offset (CFO) tracking and acquisition algorithms. And in section IV proposed a joint estimation of CFO and Channel Distortion. And Joint Estimation of Carrier Frequency Offset (CFO) and Channel Distortion Using Two Repeated Training Sequences proposed in section V.

\section{Mimo Ofdm System Model}

Multiple input multiple output (MIMO) are the use of multiple antennas at both the transmitter and receiver to improve performance of the communication system. MIMO technology has gained lots of attention in wireless communication systems because it offers significant increases in data throughput and link range without 
additional bandwidth. The combination of Multiple Input Multiple Output (MIMO) with Orthogonal Frequency Division Multiplexing (OFDM) is the best technology for the fourth generation (4G) wireless communications. However, the high data rate performance of the MIMO OFDM system is very sensitive to Carrier Frequency Offset (CFO) which introduces Inter Carrier Interference (ICI).

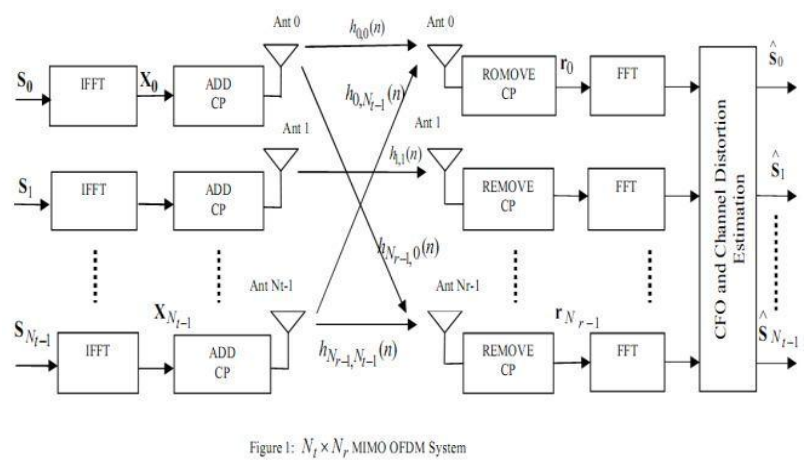

Figure: 1. Nt*Nr MIMO OFDM SYSTEM

Consequently, Carrier Frequency Offset (CFO) and Channel Distortion estimation plays a key role in MIMO OFDM systems. MIMO-OFDM system is a combination of MIMO and OFDM techniques to achieve the high spectral efficiency and increases data rate. MIMO-OFDM system transmits independent modulated signals from multiple antennas simultaneously [4]. At the receiver receives the input data, and after demodulate the data, MIMO decoding on each of sub channels extracts the data from all the transmitted antennas data on all the sub channels. From the figure $1 \mathrm{Nt}$ is the number of transmitter antennas and $\mathrm{Nr}$ is the number of receiver antennas. The MIMO OFDM system transmitter has Nt parallel transmission paths which are very similar to the single antenna OFDM system. Each and every branch channel perform serial to parallel conversion, N-point IFFT (Inverse Fast Fourier Transform), pilot insertion and cyclic prefix (CP) or cyclic extension before the final transmission. The channel encoder and modulation can also be done on each branch, but not necessary implemented jointly over each Nt branches. In MIMO OFDM system Nt different signals are transmitted simultaneously over the channel paths $(\mathrm{Nt} \times \mathrm{Nr})$ and each received signal is a combination of transmitted signal and distortion noise [5]. Firstly, the receiver must estimate and correct the possible symbol errors and frequency offsets. At the receiver Cyclic Prefix (CP) is removed and N-point Fast Fourier Transform is performed per receiver branch. In this project the estimation of channel distortion and CFO is based on the single carrier processing that implies MIMO detection has to be done per OFDM subcarrier. Therefore, the received signals of subcarrier $\mathrm{K}$ are routed to the Kth MIMO decoder to recover all the Nt transmitted data signals on the subcarrier. After the transmitted symbol the transmission (TX) antenna is combined and then outputted for the subsequent operations like demodulation and decoding.

\section{Carrier Frequency Offset (Cfo) Two Algorithms For Mimo Ofdm Systems}

This section describes about Carrier Frequency Offset estimation two algorithms for multiple input multiple output Orthogonal Frequency Division Multiplexing (OFDM) systems. Mody etal. proposed a method which uses training sequences for CFO and Chanel Distortion estimation in OFDM system. After performing the Fast Fourier Transform (FFT) of the training sequences at receiver, CFO estimation is achieved in frequency domain. Yao and Giannakis developed a low-complexity blind CFO estimator for OFDM systems, which can be applied to CFO estimation in MIMO OFDM systems. For estimation of Carrier Frequency Offset (CFO) each applied antenna must be assigned a training sequence. The training sequences are composed using repeated pseudo-noise (PN) sequence. Thus interference of inter transmit antenna is eliminated. The training sequences occupy different subcarriers in the same OFDM symbol interval from different transmit antennas. The structure of the training sequences is shown in above figure [1].

Channel is a medium through which data is to send out or signal is to pass from the source to destination. Some time a change in frequency \& wavelength of a wave is defined by user moving relative to source of wave or some time it is defined by multipath factor. So in both cases it take both concept of frequency as well as time selective. So the channels whose response is both time selective as well as frequency selective is known as doubly selective channel.

\subsection{Equalization In Carrier Transmission}

Equalization, is the process of adjusting the strength of number of frequencies within a signal. The basic \& main use of equalization is in recording of sound and reproduction but still there are many other applications in electronics and telecommunications. The equipment used to achieve equalization is called an equalizer. These 
devices strengthen (boost) or weaken (cut) the energy of specific frequency bands. In telecommunications, equalizers are used to occupy the frequency response-for instance of a telephone line-flat from end-to-end. In sound reproduction, equalization has come to mean the adjustment of frequency responses for aesthetic reasons, which usually produces a response that is not flat.

\subsection{Channel Model And Assumptions}

Taking a block transmission over an additive-noise frequency-selective channel consider in fading domain with memory $\mathrm{N}$ of single-carrier. To eliminate inter-block interference (IBI) Length of each block is $\mathrm{v}$ appended with a length $\mathrm{N}$-cyclic prefix. In hybrid-domain equalization architecture is proposed for single carrier systems which are known as decision feedback equalizer for single carrier frequency domain equalization (SCFDE-DFE), in which FD-LE is conducted as forward equalizer and time domain transverse filter is adopted as feedback equalization. It can be achieved by discarding the first $\mathrm{N}$ received symbols corresponding to the cyclic prefix. Now, out of every received $(\mathrm{N}+\mathrm{v})$ symbols, only $\mathrm{N}$ symbols are processed. The input-output relationship can be expressed in matrix form as follows:

$$
\mathrm{z}=\mathrm{Su}+\mathrm{t} \text {. }
$$

Where $\mathrm{z}, \mathrm{u}, \mathrm{t}$ are length-X blocks of received, input, and noise symbols, respectively. The NxN Channel matrix is circulate with first column equal to the channel impulse response (CIR) appended (N-v-1) by zeros. The input and noise symbols are assumed complex, zero-mean, and uncorrelated with variances. Based on the same principle, the structure of time domain noise predication decision feedback equalization is considered as the deformation of hybrid-domain equalizer. Introduces feedback filter and iterative design method into frequency domain. In the traditional SC-FDE-DFE is applied to SC-FDMA system for each single user.

\section{BLOCK FORMAT FOR PROPOSED TRANSMISSION SCHEMES.}
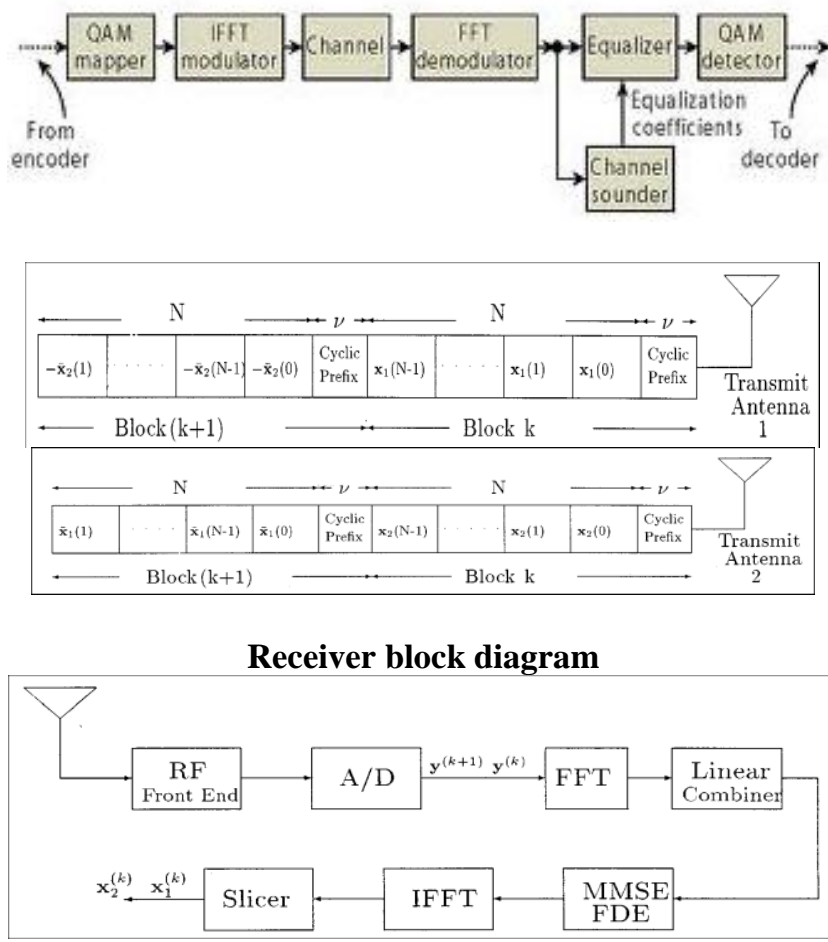

\subsection{Soft Decision Channel Estimation}

Now we try to investigate soft-decision-directed channel estimation (SDD-CE) algorithms that works in conjunction with the IFDE. Unlike conventional approaches for channel estimation, which rely on pilot symbols or hard decisions, the soft outputs of a turbo equalizer can be exploited to improve CE performance and combat error propagation. Mainly we consider a SDD time-domain CE (SDD-TDCE), which is the optimal estimator to minimize the MMSE under perfect model match assumption. However, the SDD-TDCE is computational intensive, thus not attractive for practical applications. Motivated by a significant reduction in complexity, we propose a two-stage channel estimator, the structure of which is depicted in Fig. In the first stage, per-tone softinput Kalman filtering is applied to track the channel in the frequency-domain. In the second stage, across- tone filtering is applied to refine the channel estimates. Finally, to handle the case where the channel statistics are 
unknown or time-varying, we propose a method to track the channel statistics inspired by frequency domain equalization.

In MIMO systems, a transmitter sends multiple streams by multiple transmit antennas. The transmit streams go through a matrix channel which consists of all $N_{t} N_{r \text { paths between the }} N_{t \text { transmit antennas at the }}$ transmitter and $N_{\text {receive antennas at the receiver. Then, the receiver gets the received signal vectors by the }}$ multiple receive antennas and decodes the received signal vectors into the original information. A narrowband flat MIMO system is modeled as

$y=H x+n$

where $\mathbf{y}_{\text {and }} \mathbf{x}$ are the receive and transmit vectors, respectively, and $\mathbf{H}_{\text {and }}$ nare the channel matrix and the noise vector, respectively.

Referring to information theory, the ergodic channel capacity of MIMO systems where both the transmitter and the receiver have perfect instantaneous channel state information is[12]

$C_{\text {statistical }} C_{\text {si }=\max _{Q} E\left[\log 2 \operatorname{det}\left(1+\mathrm{p} H Q H^{H}\right)\right]=E[\log 2 \operatorname{det}(1+\rho D S D)]}$

where ()$^{H}$ denotC

SNR). The optimal signal covariance $\mathbf{Q}=\mathbf{V S V}{ }^{H}$ is achieved through singular value decomposition of the channel matrix $\mathbf{U D V}^{H}=\mathbf{H}$ and an optimal diagonal power allocation matrix $\mathbf{S}=\operatorname{diag}\left(s_{1}, \ldots, s_{\min }\left(N_{t}, N_{\tau}\right), 0, \ldots, 0\right)$. The optimal power allocation is achieved through waterfilling,[16] that is $S_{i}=\left(\mu-\frac{1}{p d_{i}^{2}}\right)^{+} \quad$ fori $=1_{s \ldots \ldots \ldots, m i n}\left(N_{t_{s}} N_{y}\right)$.

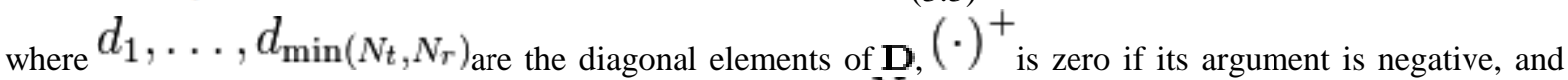
$\mu_{\text {is selected such that }} s_{1}+\ldots+s_{\min \left(N_{t}, N_{r}\right)}=N_{t}$ If the transmitter has only statistical channel state information, then the ergodic channel capacity will decrease as the signal covariance $\mathbf{Q}_{\text {can }}$ only be optimized in terms of the average mutual information as[14]

$C_{n 0-} C_{\text {gi }=E\left[\log 2 \operatorname{det}\left(1+\frac{P}{N_{\mathrm{t}}} H H^{H}\right)\right]}$

The spatial correlation of the channel has a strong impact on the ergodic channel capacity with statistical information.

If the transmitter has no channel state information it can select the signal covariance $\mathbf{Q}$ to maximize channel capacity under worst-case statistics, which means $\mathbf{Q}=1 / N_{t} \mathbf{I}_{\text {and accordingly }}$

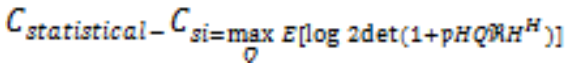

Depending on the statistical properties of the channel, the ergodic capacity is no greater than $\min \left(N_{t}, N_{r}\right)_{\text {times }}$ larger than that of a SISO system.

\subsection{Mathematical Description}

If $N$ sub-carriers are used, and each sub-carrier is modulated using $M$ alternative symbols, the OFDM symbol alphabet consists of $M^{N}$ combined symbols.

The low-pass equivalent OFDM signal is expressed as:

$V(t)=\sum_{K=0}^{N-1} e^{j 2 \pi k \frac{t}{T}}, 0 \leq t<T_{x}$

where $\left\{X_{k}\right\}_{\text {are }}$ the data symbols, $N$ is the number of sub-carriers, and $T$ is the OFDM symbol time. The subcarrier spacing of $\frac{1}{T}$ makes them orthogonal over each symbol period; this property is expressed as:

$=\frac{1}{T} \int_{0}^{T}\left(e^{j 2 \pi k_{1} \frac{t}{T}}\right)\left(e^{j 2 \pi k_{2} \frac{t}{T}}\right) d t$

$=\frac{1}{T} \int_{0}^{T} e^{j 2 \pi\left(k_{2}-k_{1}\right) \frac{t}{T}} d t=\delta_{k_{1} k_{2}}$

where $(\cdot)^{*}$ denotes the complex conjugate operator and $\delta$ is the Kronecker delta.

To avoid inter-symbol interference in multipath fading channels, a guard interval of length $T_{\mathrm{g}}$ is inserted prior to the OFDM block. During this interval, a cyclic prefix is transmitted such that the signal in the interval $-T_{\mathrm{g}} \leq t<0_{\text {equals }}$ the signal in the interval $\left(T-T_{\mathrm{g}}\right) \leq t<T$. The OFDM signal with cyclic prefix is thus: 
$V(t)=\sum_{K=0}^{N-1} e^{j 2 \pi k \frac{t}{T}}, \quad-T_{g} \leq t<T$,

The low-pass signal above can be either real or complex-valued. Real-valued low-pass equivalent signals are typically transmitted at baseband-wire line applications such as DSL use this approach. For wireless applications, the low-pass signal is typically complex-valued; in which case, the transmitted signal is upconverted to a carrier frequency $f_{c}$. In general, the transmitted signal can be represented as:

$s(t)=\Re\left\{v(t) e^{j 2 \pi k t}\right\}$

$$
\begin{gathered}
=\sum_{k=0}^{N-1} \llbracket X_{k} \llbracket \cos \left(2 \pi\left[f_{c}+{ }^{k} / T\right] t+\arg \left[X_{k}\right]\right) \\
y_{i}(N)=\sum_{n=0}^{m-1} w_{n}(N) b_{n}(N) \\
=\sum_{n=0}^{m-1} \overline{b_{n}^{*}(N) r_{i}(N)} \cdot b_{n}(N)
\end{gathered}
$$

\section{Conclusion}

In this paper, proposed new methods for the joint estimation of Carrier Frequency Offset (CFO) and Channel Distortion for MIMO OFDM systems using training sequences. The Carrier Frequency Offset (CFO) acquisition and tracking two algorithms are proposed for MIMO OFDM systems to improve the estimation accuracy. When only one OFDM block is available for training, the first method is able to give an accurate estimation of the Carrier Frequency Offset (CFO) and Channel Distortion. When two repeated training sequences are available in OFDM block, low complexity two step approach is proposed to solve the joint estimation problem. Simulation results are showed that the Mean Squared Error (MSE) of Carrier Frequency Offset (CFO) and Mean Squared Error (MSE) of Channel distortion in both proposed method and two step approach method. The simulation results are showed in two cases for comparison.

\section{References}

[1]. P. H. Moose, "A technique for orthogonal frequency division multiplexing frequency offset correction", IEEE Trans.Commun., vol. 42, no. 10, pp. 2908-2914, Oct. 2010.

[2]. Alert van Zelst and Tim C.W.Schenk, "Implementation of aMIMO OFDM-based Wireless LAN system", IEEE Trans.Signal Processing, vol. 52, No. 2, pp.483-494, Feb. 2004.

[3]. Y.-H. Chung and S.-M. Phoong, "Joint estimation of I/Qimbalance, CFO and channel response for MIMO OFDM systems" IEEE Trans. Commun., vol.58, no.5, pp.1485-1492,May 2010.

[4]. A. J. Paulraj, D. A. Gore, R. U. Nabar and H. Bolcskei, "AnOverview of MIMO Communications - A Key to Gigabit Wireless," Proc. of the IEEE, vol. 92, pp. 198-218, February2004.

[5]. X. Ma, H. Kobayashi and S. C. Schwartz, "Joint frequency offset and channel estimation for OFDM," of Global Telecommun. Conf., pp. 15-19, Dec. 2003.

[6]. Wireless LAN Medium Access Control (MAC) and Physical Layer (PHY) Specifications: High-Speed Physical Layer in the 5 GHz Band, IEEE Standard 802.11a-2005.

[7]. A. N. Mody and G. L. Stuber, "Synchronization for MIMO OFDM systems," in Proc. IEEE Globecom’01, vol. 1, Nov. 2001, pp. 509-513.

[8]. D. Huang and K. B. Letaief, "Carrier frequency offset estimation for OFDM systems using null subcarriers," IEEE Trans. Commun., vol. 54, no. 5, pp. 813-823, May 2009.

[9]. J. Tubbax, B. Come, L. V. der Perre, S. Donnay, M. Engels, H. D. Man, and M. Moonen, "Compensation of IQ imbalance and phase noise in OFDM systems," IEEE Trans. Wireless Commun., May 2005.

[10]. T. C. W. Schenk, P. F. M. Smulders, and E. R. Fledderus, "Estimation and compensation of TX and Rx I/Q imbalance in OFDMbased MIMO systems," in Proc. IEEE ICC, June 2006.

[11]. M. Ghogho and A. Swami, "Training design for multipath channel and frequency offset estimation in MIMO systems," IEEE Trans. Signal Processing, vol. 54, no. 10, pp. 3957-3965, Oct. 2006.

[12]. R. Negi and J. Cio "Pilot tone selection for channel estimation in a mobile OFDM system," IEEE Trans. Cosumer Electronics, vol. 44, No. 3, pp. 1122-1128, August 1998.

[13]. K. Lee and J. Chun, "Frequency-offset estimation for MIMO and OFDM systems using orthogonal training sequences," IEEE Trans. Veh. Technol., Jan. 2007 M. Ghogho and A. Swami, "Training design for multipath channel and frequency-offset estimation in MIMO systems," IEEE Trans. Signal Process. Oct. 2006

[14]. M. Ghogho and A. Swami, "Training design for multipath channel and frequency-offset estimation in MIMO systems," IEEE Trans. Signal Process. Oct. 2006 